\title{
High-Dose Vitamin C Exerts Its Anti-cancer Effects in a Xenograft Model of Colon Cancer by Suppressing Angiogenesis
}

\author{
Kentaro Nakanishi, ${ }^{a, b}$ Keiichi Hiramoto, ${ }^{a}$ and Kazuya Ooi*,a \\ ${ }^{a}$ Department of Pharmaceutical Science, Suzuka University of Medical Science; Suzuka, Mie 513-8670, Japan: and \\ ${ }^{b}$ Medical Nakanishi Co., Ltd.; 738-2 Idocho, Kumano, Mie 519-4324, Japan. \\ Received January 30, 2021; accepted March 18, 2021
}

Several studies have been conducted to investigate the anti-cancer effects of vitamin C (VC). However, the effect of high-dose VC administration on tumor angiogenesis remains unclear. Focusing on our high-dose $\mathrm{VC}$, our study investigated the effect of high-dose $\mathrm{VC}(4 \mathrm{~g} / \mathrm{kg})$ on vascular endothelial growth in mice with xenografts of a rectal cancer cell line referred to as Colon 26. Male mice harboring Colon 26 tumors were established, and high-dose VC solution was orally administered once daily for $14 \mathrm{~d}$. On the final day of the study, the lower limb tumor tissues and serum samples were collected and analyzed for the expression of tumor angiogenesis related proteins as well as the levels of reactive oxygen species (ROS). Oral VC administration decreased tumor volumes and increased p53 and endostatin levels. In addition, plasma and in tumor part ROS levels and tissue hypoxia inducible factor-1 $\alpha$ (HIF-1 $\alpha)$ were reduced by VC administration. In addition, the levels of vascular endothelial growth factor A (VEGFA) and vascular endothelial growth factor D (VEGFD) were decreased by VC administration. These results suggest that VC exerts its anti-cancer effects by suppressing angiogenesis.

Key words vitamin $\mathrm{C}$; endostatin; vascular endothelial growth factor (VEGF); reactive oxygen species (ROS); hypoxia inducible factor- $1 \alpha(\mathrm{HIF}-1 \alpha)$

\section{INTRODUCTION}

Vascular endothelial growth factor (VEGF) is a major regulator of angiogenesis and is involved in solid tumor carcinogenesis, infiltration, distant metastasis, and tumor angiogenesis. ${ }^{1)}$ Tumor angiogenesis plays an important role in controlling the progression of cancer. ${ }^{2)}$ There are different subtypes of VEGF with unique characteristics. VEGFA is the main subtype that efficiently induces the proliferation of endothelial cells and promotes their permeability. ${ }^{3)}$ VEGFD has a subtype with a strong angiogenic effect in advanced colorectal cancer. ${ }^{4)}$ VEGF is upregulated under various conditions associated with cancer, including the presence of various growth factors and hypoxia. ${ }^{5)}$ Transcription enhancement of the VEGF gene by hypoxia stimulation is regulated by the transcription factor hypoxia-inducing factor- $1 \alpha(\mathrm{HIF}-1 \alpha)^{6}{ }^{6}$ The production of VEGF and other growth factors by a tumor results in the formation of new vasculature in and around the tumor, but the new vascular network formed is structurally and functionally abnormal. ${ }^{5)}$ Endostatin is a C-terminal fragment of collagen XVIII and an endogenous inhibitor of angiogenesis and tumor growth, can inhibit the action of VEGF.' It has also been reported that endostatin is partially involved in tumor suppression by $\mathrm{p} 53 .{ }^{8-10)}$

Vitamin C (VC) is a water-soluble micronutrient commonly found in the diet and plays an important role as a coenzyme factor in maintaining the body. ${ }^{11)}$ Also, VC is an important antioxidant $^{12)}$ that plays a vital role in immune function. ${ }^{13)}$ It is well known that VC is essential for collagen biosynthesis. ${ }^{14)}$ Interestingly, under certain conditions $\mathrm{VC}$ can act as a prooxidant that acts to selectively suppress the growth of cancer cells. ${ }^{15)}$ The antitumor effect of VC was associated with reactive oxygen species (ROS) by administration of high-dose VC in azoxymethane + dextran sodium sulfate-induced colorectal cancer in our previous study. ${ }^{16)}$ Furthermore, in melanoma, ROS effect by administration of $\mathrm{VC}$, suggesting an antitumor effect, suggesting that ROS is important for the pathological function of high-dose VC. ${ }^{17)}$ Expression of p53 has been reported to be involved in increased intracellular ROS development, ${ }^{18)}$ and $\mathrm{VC}$ has been reported to be involved in $\mathrm{p} 53,{ }^{19)}$ but it is unclear if $\mathrm{VC}$ affect endostatin.

In this study, mice with xenografts of Colon 26 murine colorectal cancer cells were used to investigate the effects of high-dose VC on p53, VEGF and endostatin levels.

\section{MATERIALS AND METHODS}

Animals Male BALB/c mice (5 weeks old; Japan SLC, Hamamatsu, Shizuoka, Japan) were purchased. The mice were maintained in a bed net cage (made of stainless steel) under a $12-12 \mathrm{~h}$ light and dark cycle at a room temperature of $23 \pm 1{ }^{\circ} \mathrm{C}$ and humidity of $50 \pm 10 \%$. The mice were allowed free access to laboratory chow and water. At 6 weeks of age, the mice were randomly assigned to three groups $(n=6$ per group): control, Colon 26, and VC + Colon 26. All experimental protocols were approved by the animal care committee in compliance with the regulations of Suzuka University of Medical Science (Approval No. 66). The mice were euthanized by deep anesthesia with sodium pentobarbital.

The Colon 26 murine colorectal carcinoma was purchased from the Cell Resource Center for Biomedical Research (Institute of Development, Aging and Cancer, Tohoku University). ${ }^{20)} \mathrm{A}$ total of $100 \mu \mathrm{L}$ of tumor cell suspension containing $10^{5}$ cancer cells was injected subcutaneously into the right hind limbs of the mice. ${ }^{21)}$

Tumor volumes were determined using the equation (vol- 
ume $=L W^{2} / 2$ ), where $L$ is the longest dimension parallel to the skin surface and $W$ is the dimension perpendicular to $L$ and parallel to the surface.

VC Treatment We administered $1,4,8$, or $12 \mathrm{~g} / \mathrm{kg}$ of $\mathrm{VC}$ (Fuso Pharmaceutical Industries Ltd., Osaka, Japan) solution in distilled water to mice to determine the effective VC dose, and found high and equivalent effectiveness at $4 \mathrm{~g} / \mathrm{kg}$ and higher doses (data not shown). Thus, the mice in the experimental group received about $4 \mathrm{~g} / \mathrm{kg}$ of $\mathrm{VC}$ in distilled water and the control animals received distilled water. Both were orally administered once daily for $14 \mathrm{~d}^{16)}$

Preparation and Staining of Tissue Specimens On the final day of the study, the lower limb was removed under anesthesia and the tumor tissues were immunohistochemically stained with antibodies against VEGFA (1:100; Bio Legend, San Diego, CA, U.S.A.) or VEGFD (1:100; Abcam, Cambridge, U.K.). ${ }^{22}$ Briefly, the tissue samples were fixed with phosphate buffered saline (PBS) containing 4\% paraformaldehyde and then embedded in Tissue-Tek OCT Compound (Sakura Finetek, Tokyo, Japan), cut into $5 \mu \mathrm{m}$ thick sections, incubated with the primary antibodies and then incubated with fluorescein isothiocyanate-conjugated anti-rabbit and tetramethylrhodamine isothiocyanate-conjugated anti-rabbit (1:30; Dako Cytomation, Glstrup, Denmark) secondary antibodies for $2 \mathrm{~h}$ at $25^{\circ} \mathrm{C}$.

Western Blot Analysis The lower limb tumor tissues were homogenized in lysis buffer (Kurabo, Osaka, Japan) and centrifuged at $8000 \times \boldsymbol{g}$ for $10 \mathrm{~min}$. The supernatant was then removed and stored at $-80^{\circ} \mathrm{C}$ until further analysis. Western blot analysis was performed as follows with reference to the method of the previous study. ${ }^{23)}$ To briefly explain, after thawing, equal amounts of protein $(12.5 \mu \mathrm{g} /$ line $)$ were loaded onto a $4-12 \%$ BISTRIS Blot Gel (Life Technologies, Carlsbad, CA, U.S.A.) and electrophoresed at $200 \mathrm{~V}$ for $20 \mathrm{~min}$. Following separation, the proteins were transferred to a nitrocellulose membrane using the iBlot Western blotting System (Life Technologies), which was subsequently blocked with $5 \%$ skim milk at $4{ }^{\circ} \mathrm{C}$ overnight. The next day, the membranes were incubated with antibodies to p53 (1:1000; Cell Signaling Technology, Danvers, MA, U.S.A.), HIF- $\alpha$ (1:1000; Abcam), and $\beta$-actin (1:5000; Sigma-Aldrich Corp., St. Louis, MO, U.S.A.) at $25^{\circ} \mathrm{C}$ for $1 \mathrm{~h}$. Membranes were then treated with a horseradish peroxidase-conjugated secondary antibody (1:1000; Novex, Frederick, MD, U.S.A.). Immune complexes were detected with ImmunoStar Zeta reagent (Wako Pure Chemical Industries, Ltd., Osaka, Japan). Images were acquired using the Multi-Gauge Software Program ver. 3.0 (FUJIFILM, Greenwood, SC, U.S.A.).

Quantification of Endostatin and ROS Levels Blood samples were collected immediately following cardiac puncture. Plasma was separated from blood samples by centrifugation at $3000 \times \boldsymbol{g}$ for $10 \mathrm{~min}$ at $4{ }^{\circ} \mathrm{C}$, and the supernatant was used for analysis. The plasma levels of endostatin were measured using a commercial enzyme-linked immunosorbent assay kit (Endostatin: Biomedica, Vienna, Austria), according to the manufacturer's instructions. Plasma and tissue ROS levels were determined using an OxiSelect in vitro ROS/RNS assay kit (STA-347; Cell Biolabs, Inc., San Diego, CA, U.S.A.), according to the manufacturer's instructions.

Statistical Analysis All data are presented as the mean \pm standard deviation (S.D.). For comparisons be- tween test groups, by the Tukey-Kramer test was applied with $p<0.05$ considered to be a statistically significant difference.

\section{RESULTS}

Effect of VC Administration on Tumor Cell Growth Tumors were observed in mice xenografted with Colon 26 tumor cells. VC administration suppressed tumor growth. The tumor volume in the Colon $26+\mathrm{VC}$ group was reduced by $28 \%$ compared with that in the Colon 26 alone group (Fig. 1).

Effect of VC Administration on the Levels of p53, Endostatin, HIF-1 $\alpha$, and ROS p53 is a gene that drives apoptosis in cancer cells. The levels of p53 the lower limb tumor tissues were increased significantly in the Colon 26 group than in the control group. Treatment with VC further significantly increased the levels in the Colon $26+\mathrm{VC}$ group compared with that in the Colon 26 group. Plasma endostatin levels were also measured because it is partly associated with tumor suppression by p53. Similar to p53, endostatin levels were significantly increased in the Colon 26 group relative to control and further significantly increased in the Colon $26+\mathrm{VC}$ group (Figs. 2A, B). Both plasma and intratumoral ROS levels were found to be significantly increased in the Colon 26 group compared with those in the control group, but were significantly decreased in the Colon $26+$ VC group compared with those in the Colon 26 group. Similarly, the lower limb tumor tissues HIF-1 $\alpha$ levels were significantly increased in the Colon 26 group compared to the control group but were significantly decreased in the Colon $26+\mathrm{VC}$ group compared to the Colon 26 group (Figs. 2C-E).

Effect of VC Administration on the Expression of VEGFA and VEGFD the Lower Limb Tumor Tissues VEGF is a major regulator of angiogenesis. The lower limb tumor tissues expression levels of both VEGFA and VEGFD were significantly increased in the Colon 26 group compared with those in the control group, but were significantly decreased in the Colon $26+\mathrm{VC}$ group compared with those in the Colon 26 group (Fig. 3).
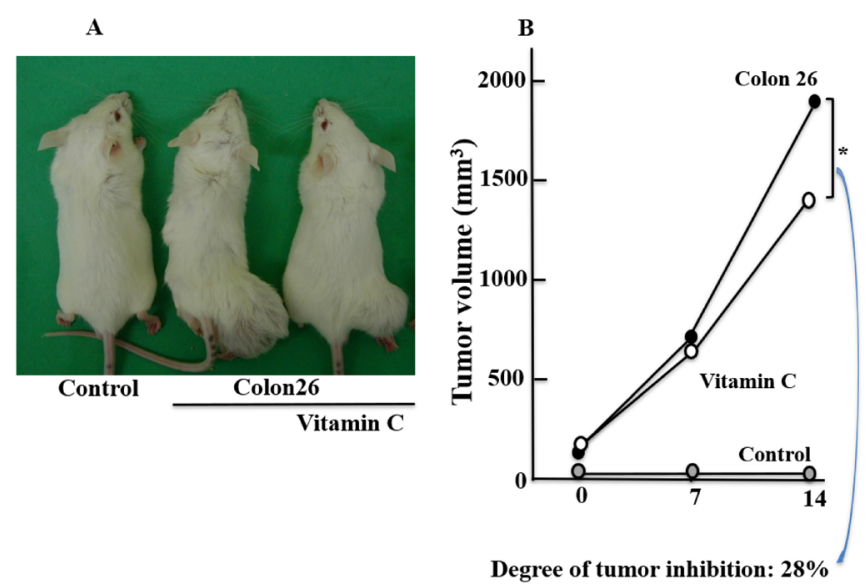

Fig. 1. Effects of VC Administration on the Growth of Colon 26 Tumors in Mice

Macroscopic view of the tumor (A) and tumor volumes (B) are shown. Data are presented as mean \pm standard deviation (S.D.) ( $n=6 /$ group). $* p<0.05$ (Tukey's post-hoc test). (Color figure can be accessed in the online version.) 

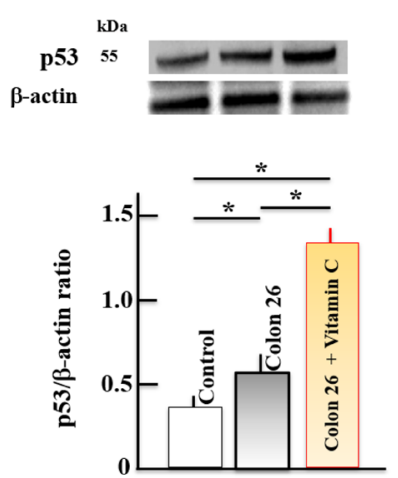

$\mathbf{A}$

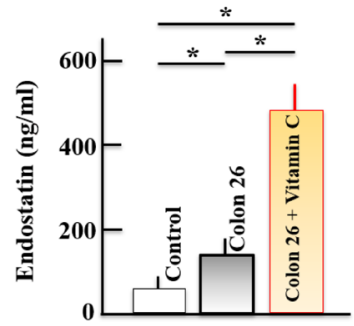

B
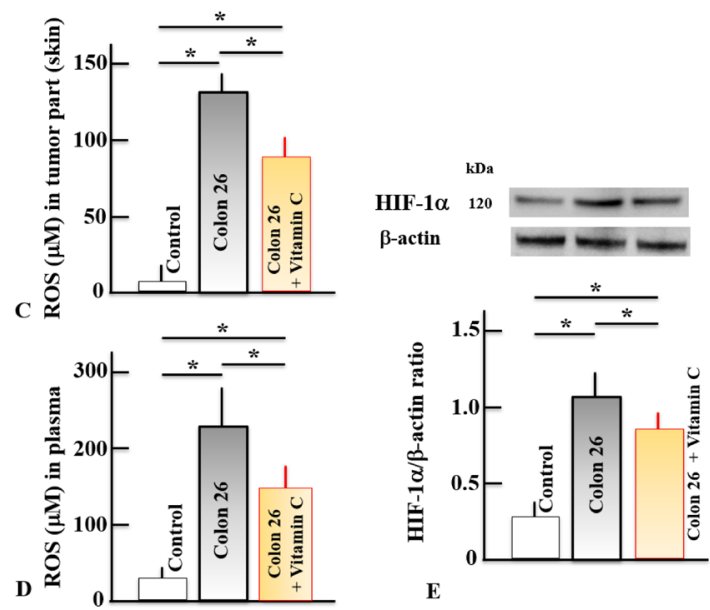

Fig. 2. Effect of VC Administration on the Lower Limb Tumor Tissues Levels of p53 (A), ROS (C), and HIF-1 $\alpha$ (E), and on Plasma Levels of Endostatin (B) and ROS (D)

Data are presented as mean \pm S.D. ( $n=6 /$ group). ${ }^{*} p<0.05$ (Tukey's post-hoc test). (Color figure can be accessed in the online version.)
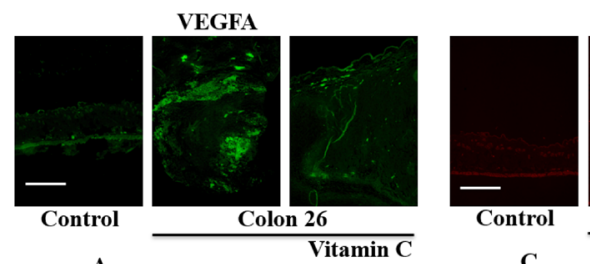

VEGFD

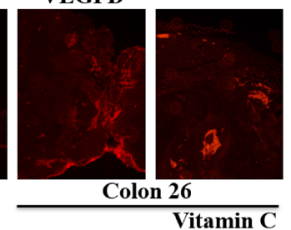

C
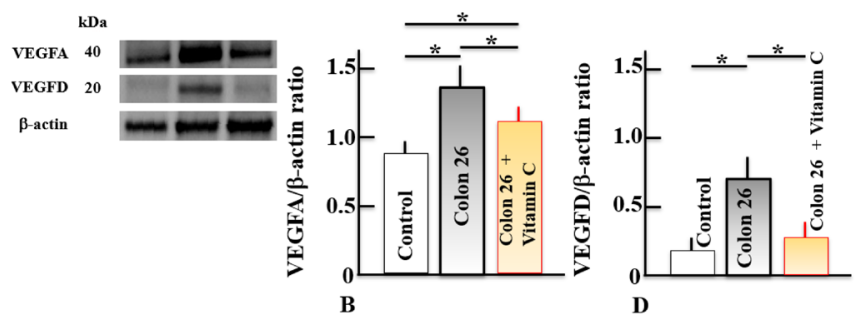

Fig. 3. Effect of VC Administration on VEGFA (A, B) and VEGFD (C, D) Levels in the Lower Limb Tumor Tissues Samples

Immunohistochemical analysis of VEGFA and VEGFD (A, C). Western blotting analysis of VEGFA and VEGFD (B, D). Data are presented as mean \pm S.D. $(n=6$ group). ${ }^{*} p<0.05$ (Tukey's post-hoc test). Scale bar $=100 \mu \mathrm{m}$. (Color figure can be accessed in the online version.)

\section{DISCUSSION}

It is well known that p53 has anti-cancer effects, it activates transcription of the $\alpha$ (II) collagen prolyl-4-hydroxylase gene. This enzyme extracellularly releases potent endogenous inhibitors of angiogenesis, such as endostatin and tumstatin, which are derived from collagen types XVIII and IV. These inhibitors suppress the angiogenesis pathway and thereby have anti-cancer effects. ${ }^{7}$ As a result of the continuous administration of $\mathrm{VC}$ for $14 \mathrm{~d}$ in mice with xenografts of Colon 26 tumor cells, tumor sizes were decreased. In addition, the expression levels of p53 and endostatin were increased and the levels of VEGFA and VEGFD were decreased. Taken together, these data suggest that VC may be involved in the p53/endostatin/ VEGF pathway. The detailed mechanism of action by which $\mathrm{VC}$ causes an increase in endostatin levels is unknown, therefore further research will be needed in the future. Furthermore, $\mathrm{VC}$ is involved in the production and maintenance of collagen and may directly affect collagen type XVIII without acting through $\mathrm{p} 53$. This aspect is currently under investigation.

$\mathrm{VC}$ has also been reported to inhibit the expression of VEGF via HIF-1 $\alpha .^{24)}$ HIF-1 $\alpha$ is hydroxylated by the oxygendependent enzyme prolyl 4-hydroxylase (PHD), but under hypoxia HIF-1 stabilizes and produces transcriptional activators. $^{25,26)}$ After that, VEGF is secreted, endothelial cells proliferate, and new blood vessels are formed. Changes in oxygen concentration also affect ROS. When ROS levels exceed the defense mechanism, ROS cause oxidative stress. Elevated ROS levels have also been reported to contribute to HIF stabilization during hypoxia and reoxygenation. ${ }^{27)}$ In mice with Colon 26 xenografts, both ROS and HIF-1 $\alpha$ levels were elevated, but both were suppressed by VC administration. It has been suggested that the suppression of ROS by VC is involved in the suppression of angiogenesis. However, it is unclear whether this effect of $\mathrm{VC}$ is affected by oxygen concentration, so further research is needed.

Acknowledgments This study was supported by JSPS KAKENHI (Grant No. 18K06802).

Conflict of Interest The authors declare no conflict of interest.

\section{REFERENCES}

1) Takahashi H, Shibuya M. The vascular endothelial growth factor (VEGF)/VEGF receptor system and its role under physiological and pathological conditions. Clin. Sci. (Lond.), 109, 227-241 (2005).

2) Zhao Y, Adjei AA. Targeting angiogenesis in cancer therapy: moving beyond vascular endothelial growth factor. Oncologist, 20, 660-673 (2015).

3) Shintani S, Murohara T. Angiogenic cytokine: VEGF. J. Jpn. Coll. Angiol., 46, 289-295 (2006).

4) Izawa N, Shitara K, Yonesaka K, Yamanaka T, Yoshino T, Sunakawa Y, Masuishi T, Denda T, Yamazaki K, Moriwaki T, Okuda $\mathrm{H}$, Kondoh C, Nishina T, Makiyama A, Baba $\mathrm{H}$, Yamaguchi $\mathrm{H}$, Nakamura M, Hyodo I, Muro K, Nakajima TE. Early tumor shrinkage and depth of response in the second-line treatment for KRAS exon2 wild-type metastatic colorectal cancer: an exploratory analy- 
sis of the randomized phase 2 trial comparing panitumumab and bevacizumab in combination with FOLFIRI (WJOG6210G). Target. Oncol., 15, 623-633 (2020).

5) Carmeliet P. VEGF as a key mediator of angiogenesis in cancer. Oncology, 69 (Suppl. 3), 4-10 (2005).

6) Forsythe JA, Jiang BH, Iyer NV, Agani F, Leung SW, Koos RD, Semenza GL. Activation of vascular endothelial growth factor gene transcription by hypoxia-inducible factor 1. Mol. Cell. Biol., 16, 4604-4613 (1996).

7) Sasaki T, Larsson H, Tisi D, Claesson-Welsh L, Hohenester E, Timpl R. Endostatins derived from collagens XV and XVIII differ in structural and binding properties, tissue distribution and antiangiogenic activity. J. Mol. Biol., 301, 1179-1190 (2000).

8) Folkman J. Tumor suppression by p53 is mediated in part by the antiangiogenic activity of endostatin and tumstatin. Sci. STKE, 2006, pe35 (2006).

9) Felbor U, Dreier L, Bryant RA, Ploegh HL, Olsen BR, Mothes W. Secreted cathepsin L generates endostatin from collagen XVIII. EMBO J., 19, 1187-1194 (2000).

10) Soond SM, Kozhevnikova MV, Townsend PA, Zamyatnin AA Jr. Integrative $\mathrm{p} 53$, micro-RNA and cathepsin protease co-regulatory expression networks in cancer. Cancers (Basel), 12, 3454 (2020).

11) Holmannová D, Koláčková $M$, Krejsek J. Vitamin $C$ and its physiological role with respect to the components of the immune system. Vnitr. Lek., 58, 743-749 (2012).

12) Frei B, England L, Ames BN. Ascorbate is an outstanding antioxidant in human blood plasma. Proc. Natl. Acad. Sci. U.S.A., 86, 6377-6381 (1989).

13) Carr AC, Maggini S. Vitamin C and immune function. Nutrients, 9 , $1211(2017)$

14) Li Y, Schellhorn HE. New developments and novel therapeutic perspectives for vitamin C. J. Nutr., 137, 2171-2184 (2007).

15) Frei B, Lawson S. Vitamin C and cancer revisited. Proc. Natl. Acad. Sci. U.S.A., 105, 11037-11038 (2008).

16) Kondo K, Sano R, Goto K, Hiramoto K, Ooi K. Administration of high-dose vitamin $\mathrm{C}$ and irinotecan ameliorates colorectal cancer induced by azoxymethane and dextran sodium sulfate in mice. Biol. Pharm. Bull., 41, 1797-1803 (2018).
17) Nakanishi K, Hiramoto K, Sato FE, Ooi K. High-dose vitamin $C$ administration inhibits the invasion and proliferation of melanoma cells in mice ovary. Biol. Pharm. Bull., 44, 75-81 (2021).

18) Ostrakhovitch EA, Cherian MG. Role of $\mathrm{p} 53$ and reactive oxygen species in apoptotic response to copper and zinc in epithelial breast cancer cells. Apoptosis, 10, 111-121 (2005).

19) An SH, Kang JH, Kim DH, Lee MS. Vitamin C increases the apoptosis via up-regulation p53 during cisplatin treatment in human colon cancer cells. BMB Rep., 44, 211-216 (2011).

20) Shimizu T, Abu Lila AS, Nishio M, Doi $Y$, Ando H, Ukawa M, Ishima Y, Ishida T. Modulation of antitumor immunity contributes to the enhanced therapeutic efficacy of liposomal oxaliplatin in mouse model. Cancer Sci., 108, 1864-1869 (2017).

21) Okamatsu A, Motoyama K, Onodera R, Higashi $T$, Koshigoe $T$, Shimada Y, Hattori K, Takeuchi T, Arima H. Folate-appended $\beta$-cyclodextrin as a promising tumor targeting carrier for antitumor drugs in vitro and in vivo. Bioconjug. Chem., 24, 724-733 (2013).

22) Yokoyama S, Hiramoto K, Koyama M, Ooi K. Skin disruption is associated with indomethacin-induced small intestinal injury in mice. Exp. Dermatol., 23, 659-663 (2014).

23) Hiramoto K, Sugiyama D, Takahashi Y, Mafune E. The amelioration effect of tranexamic acid in wrinkles induced by skin dryness. Biomed. Pharmacother., 80, 16-22 (2016).

24) Zhao L, Wang J, Zhang Y, Wang L, Yu M, Wang F. Vitamin C decreases VEGF expression levels via hypoxia-inducible factor-1 $\alpha$ dependent and independent pathways in lens epithelial cells. Mol. Med. Rep., 22, 436-444 (2020).

25) Ke Q, Costa M. Hypoxia-inducible factor-1 (HIF-1). Mol. Pharmacol., 70, 1469-1480 (2006).

26) Vetrovoy O, Rybnikova E. Neuroprotective action of PHD inhibitors is predominantly HIF-1-independent: an Editorial for 'Sex differences in neonatal mouse brain injury after hypoxia-ischemia and adaptaquin treatment' on page 759. J. Neurochem., 150, 645-647 (2019).

27) Chen R, Lai UH, Zhu L, Singh A, Ahmed M, Forsyth NR. Reactive oxygen species formation in the brain at different oxygen levels: the role of hypoxia inducible factors. Front. Cell. Dev. Biol., 6, 132 (2018). 